\title{
Nanoforensics - Dwarf detectives: A brief review
}

\author{
Mamta Malik', Rakesh Kumar Mishra, ${ }^{2, *}$, Kanwalpreet Kaur³, Sanjeev Laller ${ }^{4}$, Vijay Kumar Vijayran $^{5}$ \\ ${ }^{1,4}$ Reader, Dept. of Oral Medicine and Radiology, PDM Dental College \& Research Institute, Bahadurgarh, Haryana, ${ }^{2}$ Post \\ Graduate Resident, Dept. of Forensic Medicine \& Toxicology, Santosh Medical College, Ghaziabad, Uttar Pradesh, \\ ${ }^{3}$ Lecturer, Dept. of Conservative and Endodontics, BJS Dental College Ludhiana, Punjab, ${ }^{5}$ Dental Surgeon, Oscar Multispecialty \\ Hospital and Trauma Center, Rohtak, Haryana, India
}

*Corresponding Author:

Email: drmishrarkortho89@gmail.com

\begin{abstract}
Nanotechnology has been applied to various fields of science including electronics, engineering, physical sciences, materials sciences, biomedical sciences, pharmaceutical sciences, medical science and dentistry. Nanotechnology is becoming popular in forensics because nanoparticles can transform the bulky instruments into small chip-based system, and shorten analysis methods to make investigations, sensitive, timely, and applicable. Due to this reason nanotechnology is effectively used in the field of forensic medicine in detection and quantification of different toxic substances from various evidences like, blood, saliva, hair, teeth, vitreous humor and even from skeletal remains and fingerprint samples. So this review paper was prepared to highlight the main applications of nanotechnology in the field of forensic science.
\end{abstract}

Keywords: Nanoforensics, Nanotechnology, Forensics, Fingerprints.

\section{Introduction}

American Physicist and Nobel Laureate Dr. Richard Phillips Feynmaan in 1959 initially took innovative step towards nanotechnology and Norio Taniguchi in 1974 is the first to coin the term "Nanotechnology". The study, design, creation, synthesis, manipulation, and application of materials, devices and systems at the nanometre scale size is known as nanotechnology where the word "nano" is derived from the Greek word dwarf. A nanostructure is an object described between 1 and $100 \mathrm{~nm}$. At this size range the materials can have different and enhanced properties compared with the same material at a larger size which make them superior than other. Nanomaterials are considered advantageous and differ significantly from other materials due to the increased surface area and quantum effects. Nanomaterials can be analyzed by various techniques like electron microscopy (transmission electron microscopy, and scanning electron microscopy), atomic force microscopy, dynamic light scattering and Raman microspectroscopy. ${ }^{1-3}$

Forensic Science is a branch or specialty of science which use various techniques adapted from the natural sciences to obtain crime/criminal or other legal evidence for furtherance of law. Forensic science mainly deals with identification, evaluation, investigation of the crime, finding connections between pieces of evidence and criminals. Nano-analysis is commonly used in the nanotechnology for detection of crimes. Nanotechnology has been applied to many areas of forensic science including engineering, explosive, toxicology, pathology, serology, questioned documents, finger print, security tags, DNA typing, drug analysis, gunshot analysis, post blast analysis and odontology. This review will briefly update on methodology of nano finger prints and their role in forensic investigation along with the latest advances in the field of nanotechnology for their identification at the site of crime in solving the puzzle of various criminal cases, health hazards of nanoparticles and also briefly discuss role of nanotechnology in forensic odontology..$^{1-4}$

Nanotechnology and Finger Prints: Finger prints also known as "frictional ridge skin" are lines and swirls at the end of our fingers giving a unique pattern of ridges and valleys to each individual. They have gained utmost importance in forensics because their pattern which is unique to each individual and doesn't change throughout a person's life, even identical twins also has different fingerprints. That is why finger prints are used by the criminologists and law enforcement officers in forensic investigations. Depending upon composition fingerprints are of two types- (i) patent (visible) finger prints composed of blood, ink, paints etc. and (ii) latent (invisible) finger prints which composed of eccrine sweat and oily substances such as sebum. There are a number of optical, chemical and physical techniques available for fingerprint analysis and advanced techniques like Time-of-Flight Secondary Ion Mass Spectrometry (ToF-SIMS), Desorption Electrospray Ionization Mass Spectrometry (DESIMS), ink-jet-printed array, powdering method- most commonly used (magnetic powder, aluminum flake powder, luminescent powder and iron flake powder), chromatography, Surface Assisted Laser Desorption/ Ionization (SALDI) coupled with mass spectroscopy, Matrix Assisted Laser Desorption/ Ionization mass spectroscopy (MALDI), Raman spectroscopy, Infrared spectroscopy, including acid dyes, cyanoacrylate fuming (CA), and the evaporation of metals such as gold, zinc, and silver..$^{5-7}$ 
Latent prints are seen at all crime sites and widely found evidence in criminal investigations since they are a generalized proof of human identity. But the composition of fingerprints is dependent on number of factors like time, evaporation of volatile components of fingerprints, diffusion of fingerprints into porous surface, light, temperature and humidity etc., example more aged prints the environment might contaminant the latent fingerprints, to such environments, the fingerprints may suffer from reduced reactivity to detection when analyzed with above techniques. Print composition also varies from person to person, and is strongly affected by factors such emotional state, grooming regime, and intake of food and drugs. These inter and intra donor variability further complicates detection and interpretation of finger prints. This emergence has given birth to integration of nanotechnology with fingerprint analysis in enhancing clarity while developing the fingerprints using gold nanoparticles bristle bound with long hydrocarbon chains which form hydrophobic interactions with the sweat residues along with silver physical developer (gold and/or silver nanoparticles silver physical developer (Ag PD). The result showed that gold nanoparticles adhere to the surface with sweat residues through hydrophobic interactions which can be then developed with silver physical developer (Ag-PD), showing dark and clear impression of ridge detail with improved quality. Similarly effects of nanoparticles such as Au-NPs (gold), CdS (cadmium sulphide) and $\mathrm{ZnS}$ (zinc sulphide) for the development of latent fingerprints in order to better identify the fingerprints were also reported. Even use of hybrid nano powders can improve the visualization of latent fingerprints on multi-colored or patterned backgrounds. ${ }^{8-11}$

Moreover Worley and coworkers at Los Alamos National Lab detected latent finger prints using new method of micro-X-ray fluorescence (MXRF) which develops latent fingerprints images by detecting inorganic elements in the prints. This method has nondestructive nature of the analysis, as well as the stability of the inorganic residues, additional tests like element analysis for gunshot residue can be done, and prints can be saved up to eight months. The most commonly observed inorganic residues in fingerprints are potassium and chloride followed by silicon, calcium and aluminum. ${ }^{1,12}$

In blood stained finger prints leucomalachite green, amido black and ninhydrin chemically react with components in blood to form a dark-colored dye complex and have all been used successfully on lightcolored or transparent surfaces by their change in color. But results are beneficial only on porous surfaces. For porous and enhancement of faint blood prints on various surfaces nanoparticles have shown promising results. Age of a blood spots can be determined using nanoparticles with atomic force microscopy by testing the elasticity of blood by recording force-distance curves and calibration curve of the elasticity over time is developed. , $^{11,13}$,

Nanodentistry: Nanotechnology can be applied to forensic trace evidence analysis in cyber forensics, tape authentication and speaker identification (by identifying the nano coating material, the authentication of tapes can be determined). Nanoparticles have given satisfactory results in enhancement of PCR efficiency of creating copies of specific fragments of DNA from pulpal tissues. ${ }^{1,14}$

Health and Nanotechnology: Nanoparticles should always be open in an enclosed vessel and suitable masks should be worn, as they can easily and quickly spread in air to form aerosols which remains in environment a long time. Small size of nanoparticles after inhalation may be able to translocate from the alveolar region in the lung to the extra pulmonary organs (liver, heart, spleen, etc.) via systemic blood circulation. Central nervous system is also affected by nanoparticles deposition on the olfactory mucosa in the nasopharyngeal region and subsequent translocation via the olfactory nerve affected the whole body. Airpurifying respiratory protection system should always be followed. Hand washing should be strictly followed and hand-to-mouth activities should be avoided during handling nanoparticles. Nanoparticles should be carried in air tight suitable containers. ${ }^{9}$

\section{Conclusion}

The new methods of nanotechnology in finger print analysis made easy to unmask the new evidence in the crime cases by handling of evidence at crime scenes, its analysis in the laboratory and its presentation in the court room. Nano-forensics, a novel field of forensic science utilizes nanoparticles very effectively to develop latent fingerprints and there is still research being done in this area to improve the efficiency and performance of fingerprint detection for enhanced and reliable collection of forensic information.

\section{References:}

1. Chen YF. Forensic Applications of Nanotechnology. $J$ Chin Chemi Soci 2011;58:828-35.

2. Srividya B. Nanotechnology in Forensics and Its Application in Forensic Investigation. $J$ Pharmace Nanotech 2016;4(2):1-7.

3. Cantu AA. Nanoparticles in Forensic Science. Proc of SPIE 2008;7119:71190F-2.

4. Kaushik M, Mahendru S, Chaudhary S, Kukreti S. DNA Fingerprints: Advances in their Forensic Analysis Using Nanotechnology. J Foren Biomed 2017;8:131.

5. Hazarika P, Russell DA. Advances in fingerprint analysis. Angewandte Chemie International Edition 2012;51:352431.

6. Daluz HM. Fundamentals of Fingerprint Analysis. CRC Press. 2014.

7. Ifa DR, Jackson AU, Paglia G, Cooks RG. Forensic applications of ambient ionization mass spectrometry. Analyti Bioanalyti Chemi 2009;394:1995-2008. 
8. Lodha AS, Pandya A, Shukla RK. Nanotechnology: An Applied and Robust Approach for Forensic Investigation. Foren Res Criminol Int J 2016;2(1):00044.

9. Chaudhary J, Tailor $\mathrm{G}$ and Joshi A. A review: Nanotechnology and Forensic Science. World J Pharma and Pharmace Scie 2017;6(4):540-54.

10. Fernandes D, Krysmann MJ, Kelarakis A. Carbon dot based nanopowders and their application for fingerprint recovery. Chem Commun 2015;51(23):4902-5.

11. Prasada V, Lukoseb S, Prasada L. Emerging Forensic Applications of Nanotechnology. IJEAS 2016;2(2):42-8.
12. Worley C G; Wiltshire S S; Miller T C; Havrilla G J; Majidi V J. Detection of Visible and Latent Fingerprints Using Micro-X-ray Fluorescence Elemental Imaging. $J$ of Foren Sci 2006;51(1):57-63.

13. Sametband M, Shweky I, Banin U, Mandler D, Almog J. Application of nanoparticles for the enhancement of latent fingerprints. Chem Comm 2007;12(11):1142-4.

14. Kanaparthy A, Kanaparthy R. The Dental Role in Forensic Medicine. Iosr J Pharmac. 2013;3(3):14-7. 